\title{
DICOM format: definition and practical use in vascular medicine
}

\section{JF Uhl ${ }^{1}$}

1 M.D, FacPh, Vascular surgeon, member of the French Academy of Surgery Research director of the Unesco Chair of digital anatomy (Paris Descartes University) - www.anatomieunesco.org

submitted: Apr 1, 2020, accepted: Jun 7, 2020, EPub Ahead of Print: Aug 12, 2020, published: Aug 25, 2020

Conflict of interest: None

DOI: 10.24019/jtavr.81 - Corresponding author:Prof. Jean François Uhl, jeanfrancois.uhl@ gmail.com

(C) 2018 Fondazione Vasculab impresa sociale ONLUS. All rights reserved.

Abstract The imaging is essential in vascular medicine for diagnosis and treatment. New imaging techniques since the advent of the spiral CT scan, invented by Hounsfield and Mac Cormack in 1972, brought us a revolution in whole medicine. This has been possible thanks to the development of new standards for imaging, particularly DICOM format. The aim of this paper is to explain what is DICOM and how we use it in vascular medicine. DICOM (Digital Imaging and Communications in Medicine standards) is an universal worldwide format used for medical imaging for all vascular investigation techniques: sonography, CT (computed tomography) and MRI (magnetic resonance imaging). This format allows 3D reconstruction of the patient's anatomy and gives access to quantification of all anatomical structures of the body, in particular of the vessels. It is achieved with dedicated software called DICOM browsers. These new tools of

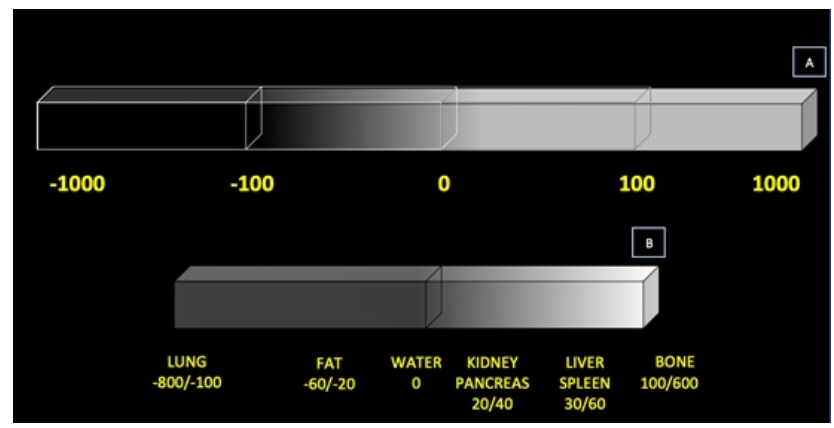

Fig. 1 - A: Hounsfield Unit scale B: Segment encompassing certain remarkable anatomical stuctures tridimensional (3D) modeling of the vessels bring us more accurate data of vascular anatomy. They make us enter in an era of new endoscopic and surgical techniques fully based on the image data, and so open the way for simulation, training and augmented reality.

Keywords DICOM, imaging, 3D modeling, 3D reconstruction, vessels-anatomy

Outline

What is DICOM file?

What is DICOM used for?

What is the content of a DICOM file?

Imaging and reconstruction modalities with DICOM

Practical use in medicine

Methodology for $3 d$ printing of the vessels

Examples of the DICOM use

\section{Introduction}

The DICOM ${ }^{1}$ is a standard of medical image and is universally used by radiologists and practitioners for the diagnostic in radiology all over the world.

Furthermore, the reconstruction of rendered volumes from DICOM bioimages has proven to be a great tool for the analysis and virtual reproduction of human anatomy. Knowing the procedure and the scope of the different tools is mandatory to lead to an accurate diagnosis, improve the surgery thanks to simulation and augmented reality techniques. 


\begin{tabular}{|c|c|}
\hline & The DICOM header \\
\hline AccessionNumber & LargestImagePixelValue \\
\hline AcquisitionDate & Manufacturer \\
\hline AcquisitionNumber & ManufacturersModelName \\
\hline AcquisitionTime & MediaStorageSOPClassUID \\
\hline BitsAllocated & MediaStorageSOPInstanceUID \\
\hline BitsStored & MetaElementGroupLength \\
\hline BodyPartExamined & Modality \\
\hline CodeMeaning & OperatorsName \\
\hline CodeMeaning & PatientID \\
\hline CodeMeaning & PatientPosition \\
\hline CodeValue & PatientsAge \\
\hline CodeValue & PatientsBirthDate \\
\hline CodeValue & PatientsName \\
\hline Columns & PatientsSex \\
\hline ContentDate & PatientsWeight \\
\hline ContentTime & PerformedProcedureStepDescription \\
\hline ConvolutionKernel & PerformedProcedureStepID \\
\hline DataCollectionDiameter & PerformedProcedureStepStartDate \\
\hline DateofLastCalibration & PerformedProcedureStepStartTime \\
\hline DeviceSerialNumber & PerformingPhysiciansName \\
\hline DistanceSourcetoDetector & PhotometricInterpretation \\
\hline DistanceSourcetoPatient & PixelData \\
\hline Exposure & PixelRepresentation \\
\hline ExposureTime & PixelSpacing \\
\hline FileMetaInformationVersion & PositionReferenceIndicator \\
\hline FilterType & ProcedureCodeSequence \\
\hline FocalSpots & ProtocolName \\
\hline FrameofReferenceUID & ReconstructionDiameter \\
\hline Gantry/DetectorTilt & ReferencedImageSequence \\
\hline GeneratorPower & ReferencedPerformed \\
\hline & ProcedureStepSequence \\
\hline HighBit & ReferencedSOPClassUID \\
\hline ImageComments & ReferencedSOPClassUID \\
\hline ImageOrientationPatient & ReferencedSOPClassUID \\
\hline ImagePositionPatient & ReferencedSOPClassUID \\
\hline ImageType & ReferencedSOPInstanceUID \\
\hline ImplementationClassUID & ReferencedSOPInstanceUID \\
\hline ImplementationVersionName & ReferencedSOPInstanceUID \\
\hline InstanceNumber & ReferencedSOPInstanceUID \\
\hline InstitutionAddress & ReferencedStudySequence \\
\hline InstitutionName & ReferringPhysiciansName \\
\hline KVP & RequestAttributesSequence \\
\hline
\end{tabular}

RequestedProcedureCodeSequence

RequestedProcedureDescription

RequestedProcedureID

RequestingService

RescaleIntercept

RescaleSlope

RotationDirection

Rows

SamplesperPixel

ScheduledProcedureStepDescription

ScheduledProcedureStepID

ScheduledProtocolCodeSequence

SeriesDate

SeriesDescription

SeriesInstanceUID

SeriesNumber

SeriesTime

SliceLocation

SliceThickness

SmallestImagePixelValue

SoftwareVersions

SOPClassUID

SOPInstanceUID

SourceImageSequence

SpecificCharacterSet

StationName

StorageMediaFile-setUID

StudyDate

StudyDescription

StudyID

StudyInstanceUID

StudyTime

TableHeight

TimeofLastCalibration

TransferSyntaxUID

WindowCenter

WindowCenterWidthExplanation

WindowWidth

$\mathrm{X}$-rayTubeCurrent

Table I - Contents of a DICOM file header

The practical use of DICOM could be very important also for telemedicine. In addition, DICOM is used also to store functional data, like blood velocity signals and spectral analysis. In this paper we will just focus on the imaging data. 


\section{I- What is a DICOM file?}

The DICOM ${ }^{1}$ Image file is a tagged image file; the file contains both an image (most of the time) and a collection of data about the image. The data in a DICOM image file is stored as a sequence of individual elements. Each element contains one item of information about the image or the image itself. DICOM elements are binary, so DICOM files cannot be viewed with a text editor.

Although each method has its own physical principles and specific equipment, all biomedical data are stored in a common format: the DICOM file.

For the digitization* of bioimages and prevent the subsequent conflicts of compatibility between files of different origin, in 1993 the American College of Radiology (ACR) in conjunction with the National Electrical Manufacturers Association of the United States (NEMA) developed and implemented the Digital Imaging and Communications in Medicine standards ${ }^{1}$ (DICOM).

These are a series of detailed specifications that describe the suitable formats and exchange of medical images mode. The core information of a DICOM file is the bioimage (.dcm) and contains inseparable data on the patient and the study performed since it has IOD (Information Object Definition) containing all the informations of the patient.

In this way it is possible to transfer and reproduce images beyond the manufacturer (Siemens Healthcare®, Philips $®$, GE Healthcare ${ }$, etc.), and even via the web (HL7 v3), for the convenience of its various users.

*Digitize: Convert or encode into digit (numbers), data or information such as an image or a document.

\section{Principle of CT scan}

It is necessary to understand what are the voxels, the Hounsfield Units and the Threshold tool.

\section{Definition of a voxel}

Just as the pixel represents the smallest element that make up a flat (2D) image, the voxel is a small cube with a side of 100 microns that make up the acquisition volume of the patient.

\section{Hounsfield Units (HU)}

It is the unit assigned to each voxel that makes up a biomedical digital image and expresses the coefficient of linear attenuation corresponding to the anatomical structure subjected to $\mathrm{X}$ radiation. For practical application, these units are subject to a scale called the Hounsfield
Scale, which is a quantitative scale that discriminates the radiodensity of human tissues. (fig 1) Thus, water corresponds to $0 \mathrm{HU}$; liver under normal conditions, an attenuation of approximately $60 \mathrm{HU}$, while lung tissue -500 $\mathrm{HU}$ and compact bone about $1000 \mathrm{HU}$.

This tool also allows to determine the colors (RGB) and the degree of opacity of each structure.

\section{Threshold tool}

The thresholding is the simplest way to achieve a segmentation of the image. It consists in the selection within the volume of voxels included between a minimum and a maximum HU value. The voxels outside these values are not displayed.

\section{DICOM standard references}

The DICOM Standard1 with its official documents can be downloaded or read online as web pages.

DICOM Browser - https://DICOM.innolitics.com/ This link is a useful website for browsing the various DICOM information objects.

\section{What is DICOM used for?}

DICOM is used in virtually all health structures ${ }^{2}$ worldwide. It ensures the interoperability of systems used to: Produce, Store, Display, Send, Query Process, Retrieve, print medical images and derived structured documents, as well as to manage the related workflow.

\section{Who needs DICOM?}

Radiologists and physicians get better access to images and reports when DICOM is in place. This allows them to make a faster diagnosis, potentially from anywhere in the world.

Patients can obtain faster and more effective care when DICOM is used to send their information through the healthcare enterprise.

Hospitals, clinics, imaging centers and specialists can make conformance to specific features of DICOM part of their purchasing requirements to ensure these tools work together across vendors to produce, manage and distribute images.

Manufacturers of imaging equipment (CT, MR, Ultrasound ...), Picture Archiving and Communication System (PACS), and peripheral equipment (workstations, 3D printers, CD importers) conform to DICOM to ensures compatibility of their equipment at every medical imaging facility. 


\section{$3 \mathrm{D}$ viewers}

Name free website

\begin{tabular}{|c|c|c|c|}
\hline HOROS & MAC & YES & https://horosproject.org/ \\
\hline OSIRIX & & NO & https://www.osirix-viewer.com \\
\hline 3DSLICER & $\mathrm{Mac} / \mathrm{pc}$ & NO & https://www.slicer.org \\
\hline MicroDICOM & $\mathrm{PC}$ & YES & http://www.microDICOM.com/downloads.html \\
\hline RadiAnt DICOM Vi & $\mathrm{PC}$ & NO & https://www.radiantviewer.com/ \\
\hline iilips DICOM Viewer & $\mathrm{PC}$ & NO & $\begin{array}{l}\text { clinical.netforum.healthcare.philips.com/global/Explore/ } \\
\text { Clinical-News/MRI/Philips-DICOM-Viewer-download- } \\
\text { version-R30-SP3 }\end{array}$ \\
\hline
\end{tabular}

Sante DICOM Viewer PC YES https://www.santesoft.com/win/sante-DICOM-viewer-

FREE pro/download.html

ORS Visual Lite $\quad$ PC $\quad$ NO http://www.theobjects.com/orsvisual/index.html

Mango Mac NO http://ric.uthscsa.edu/mango/mango.html

ORPALIS DICOM PC NO https://www.orpalis.com/labs/DICOM-viewer

viewer

Onis $\quad$ PC $\quad$ YES http://www.onis-viewer.com/ProductInfo.aspx?id=19

MiViewer PC NO https://www.millensys.com/forms/ download miviewer.php

Ginkgo CADx PC NO http://ginkgo-cadx.com/en/

MEDISP Lab DICOM PC NO http://www.bme.teiath.gr/medisp/

Viewer

Weasis

YAKAMI DICOM $\quad$ PC

downloadMEDISPDICOMViewer.htm

PC YES https://sourceforge.net/projects/dcm4che/

PC NO https://www.kuhp.kyoto-u.ac.jp/ diag rad/intro/tech/ DICOM tools.html

DICOM Viewer 2.0 PC YES https://www.robomedical.com

Open DICOM Viewer PC YES https://sourceforge.net/projects/opendicomviewer/

Agnoso PC YES http://www.e-DICOM.com/

Table II - Main DICOM viewers

\begin{tabular}{|c|c|c|c|c|}
\hline \multicolumn{5}{|c|}{$3 \mathrm{D}$ printing } \\
\hline Software & function & input format & action & output format \\
\hline HOROS $®$ & DICOM viewer & DICOM & $\begin{array}{l}\text { Extracting a 3D mesh } \\
\text { gross segmentation }\end{array}$ & obj \\
\hline MESHMIXER® & $\begin{array}{l}\text { 3D modeling } \\
\text { software }\end{array}$ & obj & $\begin{array}{l}\text { Refinement of segmentation } \\
\text { Cleaning - mesh repair }\end{array}$ & obj/mix \\
\hline CURA® & for $3 \mathrm{D}$ print & obj & Parameters for 3D printing & gcode \\
\hline
\end{tabular}




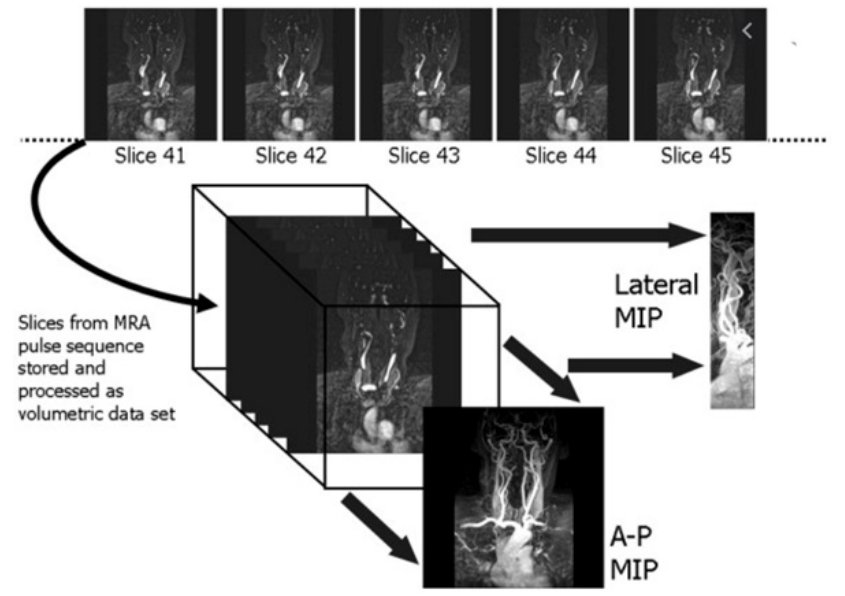

Fig. 2 - Maximum intensity projection technique (MIP)

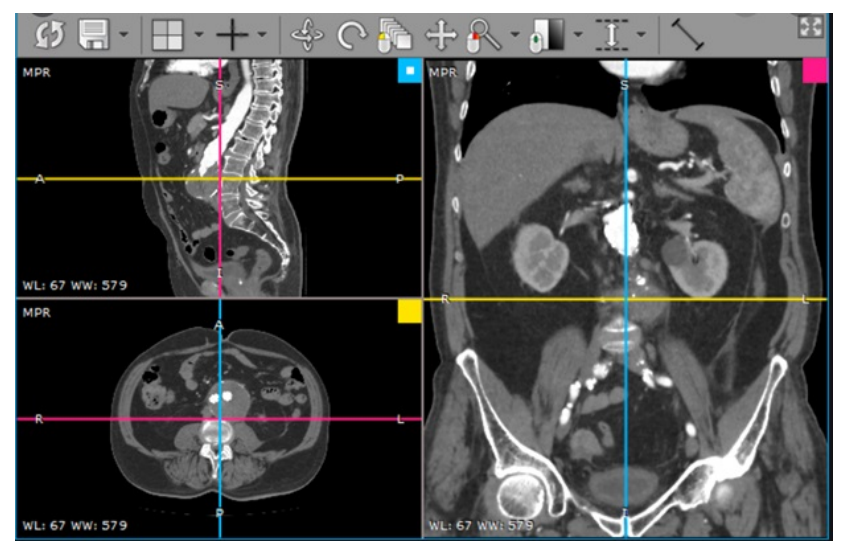

Fig. 3 - Multiplanar reconstruction technique (MPR) correspondence of the 3 planes sagittal (top left) axial (bottom left) and coronal (right)

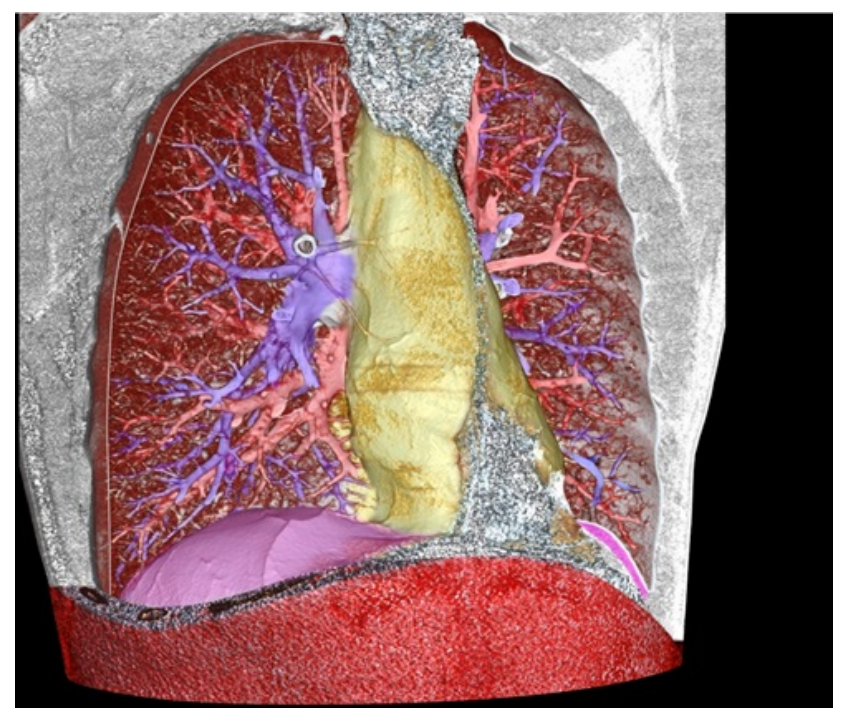

Fig. 4 - 3D direct volume rendering of the thorax (VRT)
Payers benefit from this faster and more effective process through potentially lowered cost of care.

DICOM's purpose is to meet each of these different requirements.

\section{What is the contents of a DICOM file?}

2 parts: header and data

1- the header contains all informations regarding the patient, the exam and the parameters of the following data: tag, data type and length (see table I)

2- image DATA = images under the DICOM format (512 x 512 pixels) commonly coded into on 256 bits that is 256 levels of gray. But DICOM could support higher resolution and color-coded images.

\section{II- IMAGING \& RECONSTRUCTION MODALITIES with DICOM}

It is possible to use dedicated software named DICOM browser ${ }^{3}$ (see Table II) to obtain different reconstructions of the DICOM files:

1. Maximum intensity projection (MIP) is a method for $3 \mathrm{D}$ data that projects in the visualization plane the voxels with maximum intensity that fall in the way of parallel rays traced from the viewpoint to the plane of projection (fig 2). It connects the high intensity dots of the blood vessels in three dimensions, providing an angiogram that can be viewed from any direction.

2. Multiplanar reformation or reconstruction (MPR) involves the process of converting data from an imaging modality acquired in a certain plane, usually axial, into another plane (fig 3)

3. $3 \mathrm{~d}$ reconstruction by volume rendering technique (VRT) It is a type of visualization technique which creates a three dimensional representation of data with voxels, using view-aligned texture mapping and diffuse reflection (Figure 4).

\section{III- Practical use of DICOM imaging in medicine}

$3 \mathrm{~d}$ reconstruction for patient assessment IN SURGERY and RADIOLOGY by VRT is a true revolution: More accurate morphological 3D information from new imaging techniques open the way for pre-procedure surgical planning and simulation.

3D printing from angio-CT DICOM ${ }^{4-6}$ files makes surgery safer for patients by placing real anatomy in the hands of surgeons before they go to theatre. Image-guided is also becoming mandatory to help endoscopic and miniinvasive procedures and lower the risk of complications.

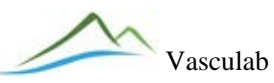


It is commonly used by orthopedic surgeons ${ }^{7}$

It is mostly true for hepatic surgery surgery as demonstrated by Soler and Marescaux ${ }^{8}$

It has also multiple applications in neurosurgery, in particular treatment of craniostenosis ${ }^{9}$, in plastic and reconstructive surgery ${ }^{10}$. In the vascular field, we commonly use today 3D models for planning, simulation, and training in vascular surgery ${ }^{11}$

Laparoscopic Quantitative 3D Endoscopy ${ }^{12}$ as well, is used for Image Guided Surgery.

$3 \mathrm{~d}$ dose delivery in radiology ${ }^{13-14}$ and Radiotherapy ${ }^{15}$ are essential to improve efficiency and avoid complications

The new dose validation system for Gamma Knife radiosurgery ${ }^{16}$ uses a DICOM-RT interface.

Working with DICOM images from CT and MRI scan data will also help Dental ${ }^{17-19}$, Medical and Veterinary practitioners ${ }^{20}$ by producing true to life $3 \mathrm{D}$ printed models of patient anatomy to inform procedures and reduce risk.

\section{IV- Methodology for 3d modeling and 3d printing of vascular models}

This could be achieved by several companies on the web providing the full service "from DICOM to print":

\section{Medimodel (https://medimodel.com)}

Think3D (https://www.think3d.in)

D2P from 3dsystems (https://www.3dsystems.com/ DICOM-to-print)

We also propose here a simple way in three steps, using the following available free software:

1- Horos ${ }^{\circledR}$ (working only on Mac computers) is a DICOM® viewer and provides a $3 \mathrm{D}$ reconstruction of the venous anatomy. It produces $3 \mathrm{D}$ vector models, also called 3D mesh, obtained by a segmentation process. Available at: https://fr.freedownloadmanager.org/Mac-OS/ Horos-GRATUIT.html (april 2020)

2- Meshmixer ${ }^{\circledR}$ is then used to clean, simplify and repair the huge $3 \mathrm{D}$ mesh file produced by Horos $囚$. Available at: https://meshmixer.updatestar.com/fr (april 2020)
- Cura ${ }^{\circledR}$ is finally used to build a "gcode" file. This will tell the 3D printer how to slice and print the $3 \mathrm{D}$ anatomical model. Available at: https:// cura.updatestar.com/fr (april 2020)

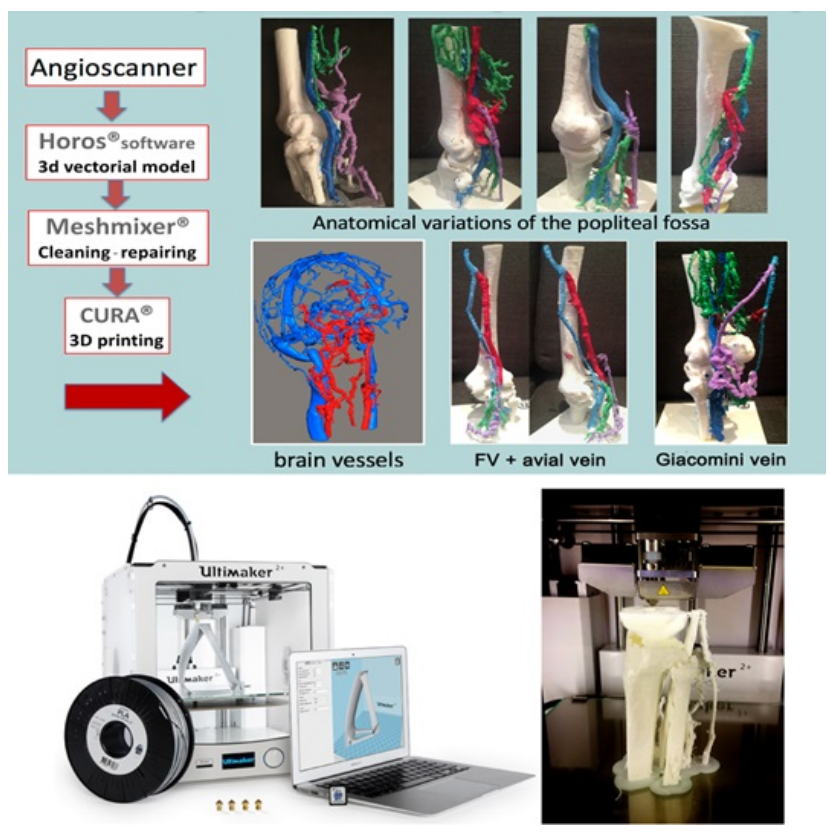

Fig. 5 - Methodology of 3D printing of educational models of venous anatomy. (workshop presented at Charing Cross 2019 and Krakow's 2019 UIP chapter meetings)

\section{V-PRACTICAL EXAMPLES of the DICOM use}

\section{1- Educational use of 3D modeling}

- CT-venography (CTV) is both a great educational tool to learn venous anatomy and a powerful research tool to improve our understanding of the venous system.

- Building with Horos ${ }^{\circledR} 3 \mathrm{D}$ animations, rotational models and journeys inside the body.

- Allows a virtual dissection of the human body ; it is a powerful teaching and learning tool for the students in human anatomy: to prepare, but not replace, cadaver dissection. (table of virtual dissection ${ }^{21}$, see figure 6)

- 3D printing of anatomical models is a great tool to study anatomical variations, which are so common in the venous network. Examples of variations of the short saphenous vein termination and the femoral vein variations are shown on figure 7. 


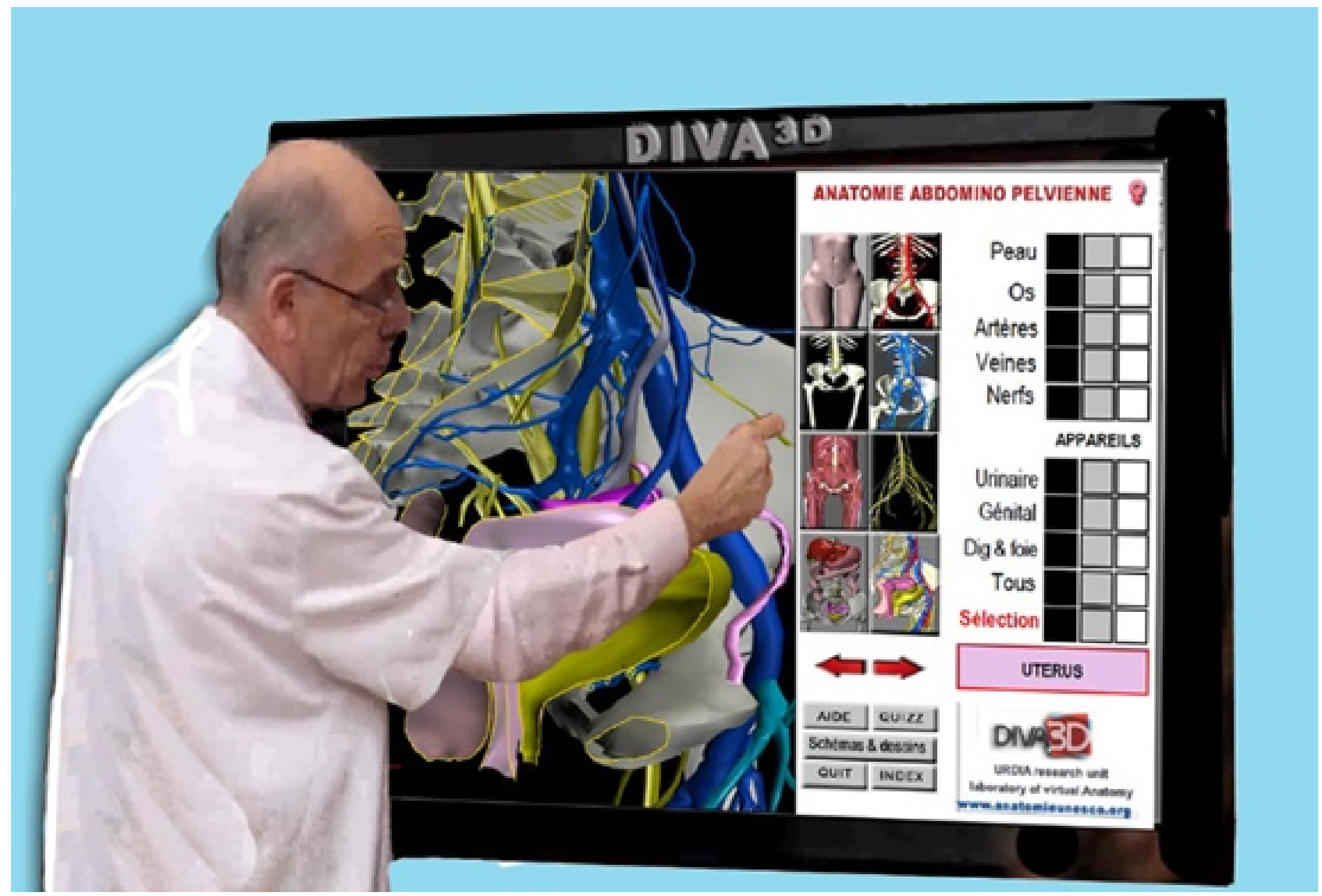

Fig. 6 - Virtual dissection table "DIVA3d" using a huge touch screen to teach and learn human anatomy.
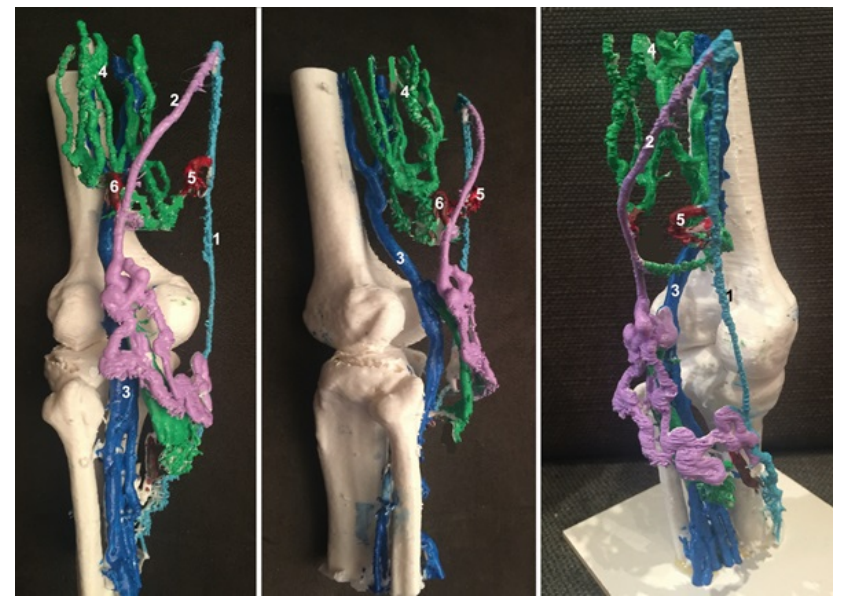

Fig. 7 - $3 d$ printed models of a patient from a CTvenography: example of a dilated Giacomini vein (in purple) Left: posterior view middle: lateral view right: posteromedial view $1=$ Great saphenous vein (in light blue) $2=$ Giacomini vein (in purple) $3=$ Popliteal vein (in dark blue) 4=Venous arcades of the semimembranosus muscle (in green) 5= Hunterian perforator vein with GSV (in red) $6=$ Thigh perforator with the Giacomini vein.

\section{2- Web communities for sharing 3d anatomical models}

For sharing 3D anatomical models, several websites are available on the web.

Some of them are totally free, and you can even download several printable $3 \mathrm{~d}$ models.

- www.embodi3d.com (biomedical 3D printing) with possibility to Automatically convert CT scans into 3D Printable Models for Free with democratiz3D®.

- NIH 3Dmodels.com show a large collection of 3D models of vascular cardiac pathology.

Other web solutions propose to host your models: Sketchfab®. (www.sketchfab.com.)

You have to subscribe to buy or sell your own collection of $3 \mathrm{~d}$ models. You can include labels of the structures (fig 8) and display the model in Virtual Reality mode. 


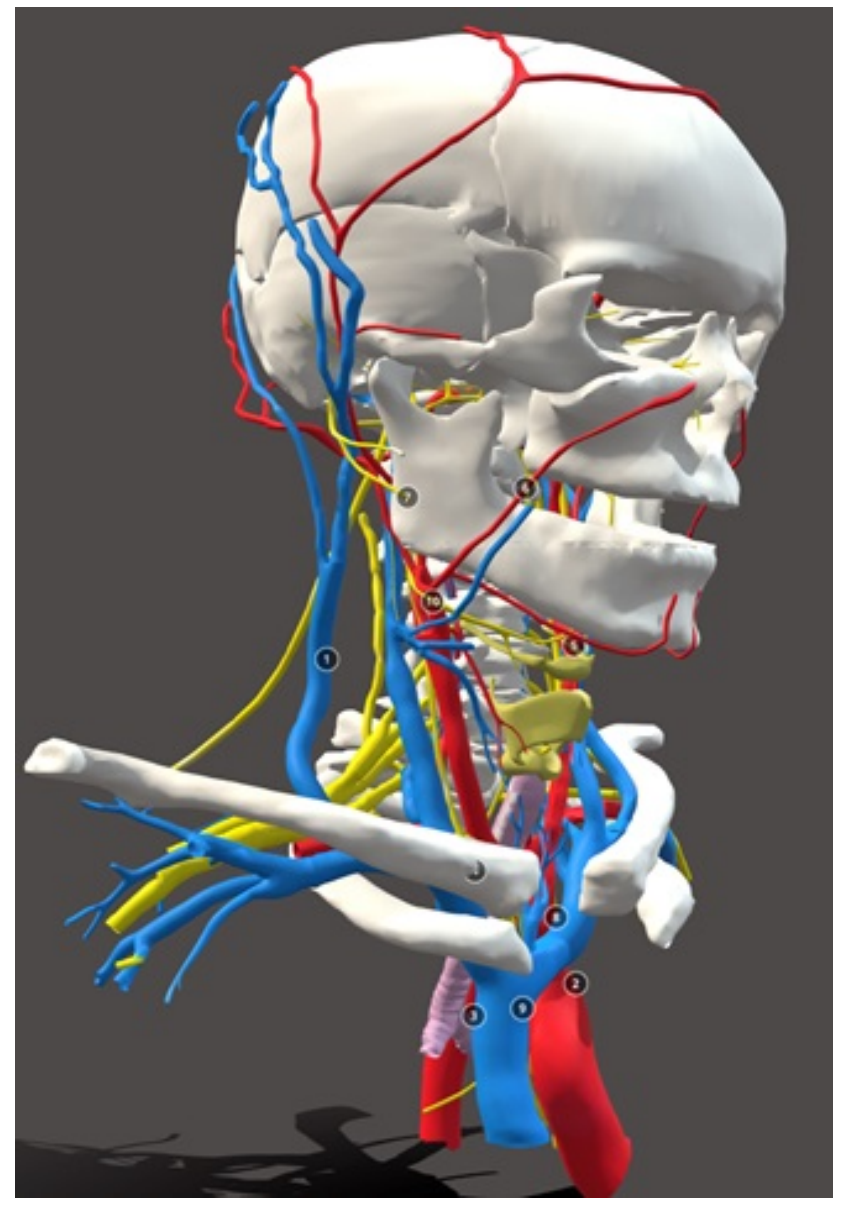

Fig. 8 - Display of the interactive 3D model of the head \& neck with labels using Sketchfab® (available at: https:// skfb.ly/6QYxZ)

\section{Conclusion}

DICOM format is a worldwide format of imaging in medicine, making possible to build realistic 3D models of human anatomy, especially in the field of vascular pathology.

The new tools of 3D modeling constitute a true revolution in educational anatomy and for clinical applications in the case of complex vascular anatomy.

It is also the future of surgery providing an accurate information about the vascular anatomy of each patient. The modern surgery has to be an image-guided surgery for an elective and more limited ablation of organs (segmentectomy) and for less complications.

\section{References}

1) Available at http://DICOM.nema.org/standard.html at the date of April 2020.

2) W D Bidgood, Jr, S C Horii. Introduction to the ACR-NEMA DICOM standard. Radiographics 1992; 12: 345-55
3) Kayhart D. Using an Existing DICOM Infrastructure to Enhance the availability, Quality, and Efficiency of Imaging Throughout the Healthcare Enterprise. J Digit Imaging. 2019 Feb;32(1):75-80

4) Ganguli A; Pagan-Diaz GJ; Grant L; Cvetkovic C; Bramlet M; Vozenilek J; Kesavadas T; Bashir R. 3D printing for preoperative planning and surgical training: a review. Biomed Microdevices (2018).

5) Tack P; Victor J; Gemmel P; Annemans L. 3D-printing techniques in a medical setting: a systematic literature review. BioMed Eng OnLine (2016) 15:115

6) Uhl J.F modeling and $3 \mathrm{~d}$ printing of the veins from ctvenograms Phlebolymphology $2020 \mathrm{~N}^{\circ} 100$ (in press)

7) Hung, C. C. et al. Conventional plate fixation method versus pre-operative virtual simulation and three-dimensional printingassisted contoured plate fixation method in the treatment of anterior pelvic ring fracture. Int. Orthop. 43, 425-431, https://doi.org/10.1007/ s00264-018-3963-2 (2019).

8) L. Soler, S. Nicolau, P. Pessaux, D. Mutter, J. Marescaux, Augmented reality in minimally invasive digestive surgery, Pediatric Digestive Surgery 2017, Mario Lima Editor, Springer, 421-432

9) Baskaran, Vivek, et al. "Current applications and future perspectives of the use of $3 \mathrm{D}$ printing in anatomical training and neurosurgery." Frontiers in neuroanatomy 10 (2016): 69Lo Presti G, Carbone M, Ciriaci D, Aramini D, Ferrari M, Ferrari V. Assessment of DICOM Viewers Capable of Loading Patient-specific 3D Models obtained by Different Segmentation Platforms in the Operating Room. J Digit Imaging. 2015 Oct;28(5):518-27. doi: https://doi.org/10.1007/ s10278-015-9786-4.

10) Shimizu F, Uehara M, Oatari M, Kusatsu M. Threedimensional visualization of the human face using DICOM data and its application to facial contouring surgery using free anterolateral thigh flap transfer. J Plast Reconstr Aesthet Surg. 2016 Jan;69(1):e1-4. doi: https://doi.org/10.1016/j.bjps.2015.07.025.

11) Moglia, A., Di Franco, G., Morelli, L. Use of 3D models for planning, simulation, and training in vascular surgery. Updates Surg 71, 185-186 (2019). https://doi.org/10.1007/s13304-019-00636-1

12) Penne, Jochen et al. "Laparoscopic Quantitative 3D Endoscopy for Image Guided Surgery.” Bildverarbeitung für die Medizin (2010).

13) Suliman II. Estimates of Patient Radiation Doses in Digital Radiography Using DICOM Information at a Large Teaching Hospital in Oman. J Digit Imaging. 2019 Mar19. doi: https://doi.org/10.1007/ $\underline{\text { s10278-019-00199-y }}$

14) Karambatsakidou A, Omar A, Fransson A, Poludniowski G. Calculating organ and effective doses in paediatric interventional cardiac radiology based on DICOM structured reports - Is detailed examination data critical to dose estimates? Phys Med. 2019 Jan;57:17-24. doi: https://doi.org/10.1016/j.ejmp.2018.12.008.

15) Griffin KT, Mille MM, Pelletier C, Gopalakrishnan M, Jung JW, Lee C, Kalapurakal J, Pyakuryal A, Lee C. Conversion of computational human phantoms into DICOM-RT for normal tissue dose assessment in radiotherapy patients. Phys Med Biol. 2019 Jul $5 ; 64(13)$

16) Choi HJ, Chung HT, Sohn JW, Min CH. Independent dose validation system for Gamma Knife radiosurgery, using a DICOM-RT interface and Geant4. Phys Med. 2018 Jul;51:117-124. 
17) Burgess J. Digital DICOM in Dentistry. Open Dent J. 2015 Jul 31;9:330-6

18) Almukhtar A, Khambay B, Ayoub A, Ju X, Al-Hiyali A, Macdonald J, Jabar N, Goto T. "Direct DICOM Slice Landmarking" A Novel Research Technique to Quantify Skeletal Changes in Orthognathic Surgery. PLoS One. 2015 Aug 7;10(8):e0131540.

19) Apostolakis D, Michelinakis G, Kourakis G, Pavlakis E. Accuracy of triangular meshes of stone models created from DICOM cone beam CT data. Int J Implant Dent. 2019 May 8;5(1):20
20) Brühschwein A, Klever J, Hoffmann AS, Huber D, Kaufmann E, Reese S, Meyer-Lindenberg A. Free DICOMViewers for Veterinary Medicine : Survey and Comparison of Functionality and User-Friendliness of Medical Imaging PACSDICOM-Viewer Freeware for Specific Use in Veterinary Medicine Practices. J Digit Imaging. 2019 Mar 11. doi: https://doi.org/10.1007/ s10278-019-00194-3.

21) http://www.diva3d.net 